\title{
A quasi-randomized group trial of a brief alcohol intervention on risky single occasion drinking among secondary school students
}

\author{
Gerhard Gmel · Vigeli Venzin · Katrin Marmet • \\ George Danko · Florian Labhart
}

Received: 16 December 2011/Revised: 26 July 2012/Accepted: 2 October 2012/Published online: 23 October 2012

(C) Swiss School of Public Health 2012

\begin{abstract}
Objectives To show the effectiveness of a brief group alcohol intervention. Aims of the intervention were to reduce the frequency of heavy drinking occasions, maximum number of drinks on an occasion and overall weekly consumption.

Methods A cluster quasi-randomized control trial (intervention $n=338$; control $n=330$ ) among 16- to 18-yearold secondary school students in the Swiss Canton of Zürich. Groups homogeneous for heavy drinking occasions $(5+/ 4+$ drinks for men/women) consisted of those having medium risk (3-4) or high risk $(5+)$ occasions in the past
\end{abstract}

G. Gmel $(\bowtie) \cdot$ F. Labhart

Research Department, Addiction Switzerland,

Avenue de Ruchonnet 14, P.O. Box 870,

1001 Lausanne, Switzerland

e-mail: ggmel@addictionsuisse.ch

G. Gmel

Alcohol Treatment Center, Lausanne University Hospital,

1011 Lausanne, Switzerland

G. Gmel

Centre for Addiction and Mental Health, 33 Russell Street, Toronto, ON M5S 2S1, Canada

G. Gmel

University of the West of New England, Frenchey Campus, Coldharbour Lane, Bristol BS16 1QY, UK

V. Venzin · K. Marmet

Cantonal Office for Secondary Education Zürich, 8090 Zurich, Switzerland

G. Danko

Department of Psychiatry, University of California, La Jolla, San Diego, CA 92093-0866, USA
30 days. Groups of 8-10 individuals received two 45-min sessions based on motivational interviewing techniques.

Results Borderline significant beneficial effects $(p<0.10)$ on heavy drinking occasions and alcohol volume were found 6 months later for the medium-risk group only, but not for the high-risk group. None of the effects remained significant after Bonferroni corrections.

Conclusions Group intervention was ineffective for all atrisk users. The heaviest drinkers may need more intensive treatment. Alternative explanations were iatrogenic effects among the heaviest drinkers, assessment reactivity, or reduction of social desirability bias at follow-up through peer feedback.

Keywords Group randomized trial .

Brief alcohol intervention .

Risky single occasion drinking · Secondary school students

\section{Introduction}

Alcohol use by adolescents and young adults is one of the most costly and largest risk factors of mortality and morbidity in established market economies (Rehm et al. 2006). In developing countries, other risk factors such as undernutrition and water hygiene are more important (Ezzati et al. 2004). 'Risky single occasion drinking' (RSOD, also called binge drinking or alcohol use leading to intoxication) is a main factor associated with many consequences such as injuries, unprotected sexual activities, and unfavorable academic development among young people (Hingson et al. 2005).

Brief intervention is a cost-effective, individual-centered preventive strategy with 1-3 short sessions (Babor et al. 2010), often based on techniques of motivational interviewing 
(MI, Miller and Rollnick 2002). Avoiding confrontation and argumentation, this approach accepts young people as selfdetermined individuals without lecturing them or declaring ultimatums, and provides an atmosphere for self-directed behavior change (Tevyaw and Monti 2004).

Research on brief alcohol interventions (BAI, Babor and Higgins-Biddle 2001) has shown some promising (Larimer et al. 2004; Tevyaw and Monti 2004) though equivocal (Handmaker et al. 1999; Murphy et al. 2004) findings among adolescents and young adults. However, few BAI studies have focused on RSOD (Kaner et al. 2007), and findings are inconclusive (Whitlock et al. 2004). The present study looks at the effectiveness of BAI delivered in two 45-min sessions focusing on RSOD, commonly defined as drinking 5 or more drinks on an occasion for men, and 4 or more drinks for women (Gmel et al. 2011).

Despite its potential, individual-centered BAI with oneon-one counseling is costly and time-consuming. The present study describes a group approach where each counselor conducts BAI simultaneously with several individuals. Advantages of group interventions are direct feedback through peers (LaBrie et al. 2007), strengthening of behavior change through reinforcement by group members (Foote et al. 1999), and bolstering autonomous motivation resulting in stronger individual change than is found in personal consultations (Ryan et al. 1995). However, a meta-analysis of MI-based group interventions suggests less efficacy in group interventions compared with personal consultations (Lundahl and Burke 2009), and McCambridge et al. (2011) recently found no significant effects from MI classroom sessions.

Group BAI sessions have been used, but were commonly not MI-oriented, not designed for younger individuals, and not very brief (often around 10 sessions, e.g. Bradley et al. 2007; Engle et al. 2010), making them impractical in most school settings. There were some MI-based group approaches designed for younger audiences and consisting of fewer (4-6) sessions (Fields 2006; D’Amico et al. 2010), but results were mixed. A few of the reviewed brief (3 or less sessions) group interventions on alcohol use by adolescents and young adults that showed small beneficial effects (Schmiege et al. 2009) commonly used no control group designs. One study (LaChance et al. 2009) found alcohol use reductions after 3 and 6 months, but other research showed short-term effects that disappeared after 6 months (LaBrie et al. 2009; Bachmann 1999).

An objective of the present study was to test a brief group intervention that can be directly implemented in the naturalistic setting of schools, providing information on effectiveness. It was important to conduct the intervention without using additional resources than those provided by the schools. The aim was to increase external validity for the intervention and not the internal validity as in many efficacy trials, where additional resources such as space outside of schools, personnel for screening, assessment and data collection are used.

\section{Methods}

Intervention design

The intervention was designed for groups of 8-10 students, grades 10-13, in secondary schools (i.e. vocational schools and gymnasiums preparing for high schools and universities) within the Zürich canton. It consisted of two 45-min intervention sessions (see Table 1 for details) based on MI techniques (Miller and Rollnick 2002) and BAI strategies suggested by Babor and Higgins-Biddle (2001).

Counselors were 12 experienced collaborators from the alcohol and drug help information centers of Winterthur, Zürich City and Zürich Oberland. All counselors have conducted brief interventions before, and they were familiar with MI principles, which were rehearsed during a half-day course prior to intervention.

Baseline assessment took place between August 2008 and March 2009. Intervention sessions were held 2-3 weeks later, with 6-month follow-ups occurring between February 2009 und November 2009.

\section{Intervention objectives}

The three objectives were to reduce an individual's RSOD frequency, maximum number of drinks on a single occasion, and overall weekly consumption at 6 months.

\section{Intervention outcomes}

The primary outcome measure was the frequency of RSOD occasions, where one occasion was defined as $4+$ drinks for females and $5+$ drinks for males. Frequencies were measured on an 8-point scale ranging from never to 12 or more occasions. Midpoints of categories were used (e.g. 3.5 for 3-4 occasions, and 13 for 12 or more occasions).

Secondary outcome measures referring to the past 30 days were the total number of standard drinks in a typical week (Monday-Sunday) and the maximum number of drinks on a single occasion. Examples of standard drinks containing $10-12 \mathrm{~g}$ of ethanol were provided for beer, wine, spirits, alcopops and cocktails (e.g., Whiskey-Cola, Caipirinha), along with conversion values (e.g. 3 cans of 0.51 beer $=6$ standard drinks).

Sample size and power considerations

International school surveys revealed design effects of about 1.2 (Hibell et al. 2003; Roberts et al. 2004), representing an 
Table 1 Content, aims, and description of intervention

\begin{tabular}{|c|c|c|}
\hline Stage of interview guideline & Aim & Exemplary description \\
\hline 1. Defining the issue for students & $\begin{array}{l}\text { To define problematic alcohol use } \\
\text { (particularly RSOD) as a help } \\
\text { for future decision-making } \\
\text { about alcohol use }\end{array}$ & $\begin{array}{l}\text { Weight was placed not to demonize alcohol use in general, but } \\
\text { to think about potential future goals in using alcohol in a } \\
\text { moderate way, by avoiding heavy use and potential } \\
\text { consequences of heavy use, including heavy use in } \\
\text { particularly hazardous situations (e.g. walking home alone at } \\
\text { nights, situations with potential for violence, heavy alcohol } \\
\text { use in traffic) }\end{array}$ \\
\hline 2. Discussing drinking behavior & $\begin{array}{l}\text { Open not confrontational } \\
\text { discussion of alcohol use in this } \\
\text { age group. The metabolism of } \\
\text { alcohol and effects on the body } \\
\text { and brain were explained in } \\
\text { relation to blood alcohol } \\
\text { concentrations }\end{array}$ & $\begin{array}{l}\text { Examples for calculating peak blood alcohol concentrations } \\
\text { were given, using examples of quantities derived from the } \\
\text { baseline assessment. The time of degradation of alcohol in } \\
\text { the body was discussed and what this may mean as regards } \\
\text { aftereffects or hangovers the next day. Experienced } \\
\text { consequences of aftereffects were discussed in the group }\end{array}$ \\
\hline 3. Raising ambivalence & $\begin{array}{l}\text { Discussion of pros and cons of } \\
\text { alcohol use (particularly RSOD) } \\
\text { using perceived pros and cons } \\
\text { raised in the discourse }\end{array}$ & $\begin{array}{l}\text { Feedback was given on the main risk factors such as injuries, } \\
\text { violence, or unprotected sex using available statistics in } \\
\text { Switzerland. Counselors informed about potential for } \\
\text { developing dependence and explained development of } \\
\text { tolerance towards the effects of alcohol }\end{array}$ \\
\hline 4. Discussion of discrepancies: & $\begin{array}{l}\text { To guide students in perceiving } \\
\text { discrepancies between positive } \\
\text { connotations of behavior and } \\
\text { experienced consequences }\end{array}$ & $\begin{array}{l}\text { Counselors reflected discrepancies raised by students to } \\
\text { provoke reactions by those, e.g. to drink to have fun and } \\
\text { vomiting or having a hangover the next day, or to drink to } \\
\text { socialize and remembering shameful situations the next } \\
\text { morning }\end{array}$ \\
\hline 5. Giving advice & $\begin{array}{l}\text { Discussion of alcohol use } \\
\text { guidelines (not more than } 3 / 4 \\
\text { drinks for girls/boys on an } \\
\text { occasion) and the perception of } \\
\text { those by students; discussion of } \\
\text { pros and cons to stay with } \\
\text { drinking guidelines }\end{array}$ & $\begin{array}{l}\text { Examples of lower body weight to explain the benefit from } \\
\text { staying below adult guidelines were given; } \\
\text { recommendations to reduce alcohol use by } 50 \% \text { as a goal } \\
\text { for very heavy drinking students were given. Examples of } \\
\text { relative risks for consequences for alcohol use over and } \\
\text { above the corresponding guidelines were given }\end{array}$ \\
\hline
\end{tabular}

Zurich, Switzerland, 2008-2009

approximate Intra Class Coefficient (ICC) of 0.01. Generally, ICCs are smaller than 0.10 (Bland 2000). We based the a priori power calculations on ICCs of 0.05 . As no information on effect sizes for secondary and vocational school students could be found in the literature, we drew on a pilot study pointing to approximately $50 \%$ of students at risk, i.e. having more than two RSOD in the past 30 days (Nelson et al. 2005). To achieve power at 0.90 with a significance level of 0.05 in our study, a sample size of 49 intervention classes and 49 control classes was needed (i.e. a total of 1,940 students at 20 per class) to show a $20 \%$ difference between intervention and control groups at follow-up (Bertholet et al. 2005; Kaner et al. 2007). Power calculations including cluster sampling were also conducted for $t$ tests on RSOD and mean number of drinks, which resulted in a need for $<49$ clusters described above. These computations were done with Pass 2008 (Hintze 2008) software, but no program was available to perform power estimations for regression analysis including cluster sampling directly. For standard logistic regression assuming a conservative OR of 1.5 and 3 independent variables (intervention, age and sex) with an assumed $R^{2}$ of $0.2,380$ students would be needed. With an assumed design effect of 1.2, this meant 456 students. Fewer individuals would be needed for regressions and analysis of variance for continuous measures. Using a conservative estimate, we planned to include 60 classes each for intervention and control conditions.

Study population, sampling and quasi-randomization

Since obtaining permission from teachers and school principals is time consuming, the largest of the 43 schools in the canton were contacted first. Selection stopped after the intended number of 120 classes was approximated. Three schools in the city of Zürich and six in rural areas provided 118 classes, but five classes withdrew their initial consent because of other priorities such as urgent exams, i.e. $113(95.7 \%)$ participated. Schools generally offered full participation (i.e. primary sampling units were schools), but only classes with students still in school at follow-up were included.

Four of the nine schools participated with full research protocol compliance, providing 78 classes for 
randomization to intervention and control conditions. Three schools agreed to the condition that all their classes receive an intervention; two others agreed only to the condition that all their classes were controls. Forty classes from these five schools were included in the study.

Only classes of the first four schools could be used for random allocation, and a random number between zero and one was generated for each class. Those with the highest numbers were assigned to the intervention condition and those with the lowest numbers to the control condition. The number of intervention and control classes was chosen to match the total number of intervention and control classes across all nine schools, including the five schools furnishing only control or intervention classes. Randomization was also stratified to match the three school types (high schools and vocational schools with either mercantile or industrial-commercial apprenticeship specializations). Across all nine schools, the three types had nearly equal ( \pm 1 class) representation under the intervention and control conditions.

According to baseline drinking patterns, homogeneous groups of 8-10 students were formed for receiving the intervention, i.e. "medium risk", with 3 or 4 RSOD occasions during the last 30 days, and "high risk", with 5 or more RSOD occasions. Intervention students came from the same or parallel classes within schools. Those not at risk ( $<3 \mathrm{RSOD})$ received a lecture on general health issues. Controls were students from control classes with the same definition of risks (see Fig. 1).

There were no exclusion criteria. Questionnaires were distributed with blank envelopes that students could return sealed even if the entire questionnaire was unanswered. Ethical approval was obtained from the responsible department in the Mittelschul- und Berufsbildungsamt des Kantons Zürich (Cantonal Office for Secondary Education Zürich, COSEZ). At the end of the consultation, students were encouraged to continue talking about alcohol use, and addresses of help and information centers were provided, as were business cards of counselors in the event that continued one-to-one counseling was wanted. Since baseline, only seven intervention students and six controls initiated any helpdesk or psychological counseling services. Given the small and nearly equal numbers, this should not have affected the results.

\section{Implementation and blinding}

Throughout the study, there was a strict separation of the trial evaluators (GG, FL) and those providing the intervention. Whereas the enrollment of schools was done by COSEZ, the quasi-randomization and allocation of students to groups was completed by the evaluators, without any contact with counselors or school officials.
At baseline, a 5-10 min questionnaire of seven items was distributed in classes. One-to-one codes (a combination of own birthday and first name of a grandmother) were created by each student and placed with the completed questionnaire in sealed envelopes which were opened later at the trial evaluation site. There, lists of the codes with allocations to classrooms were provided to school officials, showing where the interventions or the general health lectures took place. Officials were blinded to the content of the corresponding classroom sessions. Similarly, schools with random allocation were blinded to randomization of classes, and schools and counselors were blinded to allocation of students into groups.

\section{Statistical methods}

To test for equivalence at baseline between intervention and control individuals, Chi-squared tests for categorical variables (sex, and at-risk status) and $t$ tests for continuous variables (frequency of RSOD, maximum number of drinks on an occasion and total number of drinks in a typical week) were used. For the analysis of attritors (those lost to follow-up), logistic regressions for categorical, and analysis of variance for continuous measures were used. Main effects in the attrition analysis show whether attrition is associated with sex (logistic regression analysis) or with age (analysis of variance), and the interaction effects show whether attrition was differential among intervention and control groups (attrition bias).

Analysis of baseline equivalence between intervention and control individuals or between attritors and non-attritors conservatively did not account for cluster sampling. This strategy increased the probability of finding nonexistent differences (Type 1 error). Baseline equivalence and lack of attrition bias was assumed for tests with $p>0.10$ (Petitti 2000).

Multiple linear regressions were used in the main analyses to show outcome differences between intervention and control groups; of interest were frequency of RSOD, total number of drinks in a typical week and maximum number of drinks on a single occasion. Intention to treat analysis with the last value carried forward was used. Models accounted for cluster effects (STATA version 11 software with the "robust cluster" option) and were adjusted for age and sex. No variables were transformed, since sample sizes were sufficiently large to justify analysis of skewed variables (Lumley et al. 2002), and less skewed change scores were used. Main outcomes were considered significant at $p<0.05$.

Multiple regressions were used in ancillary intention to treat analyses on the same alcohol outcomes as in the main analyses, separately for the medium and high risk groups and for both sexes. Significance was set at $p<0.10$ for 
Fig. 1 Flow diagram

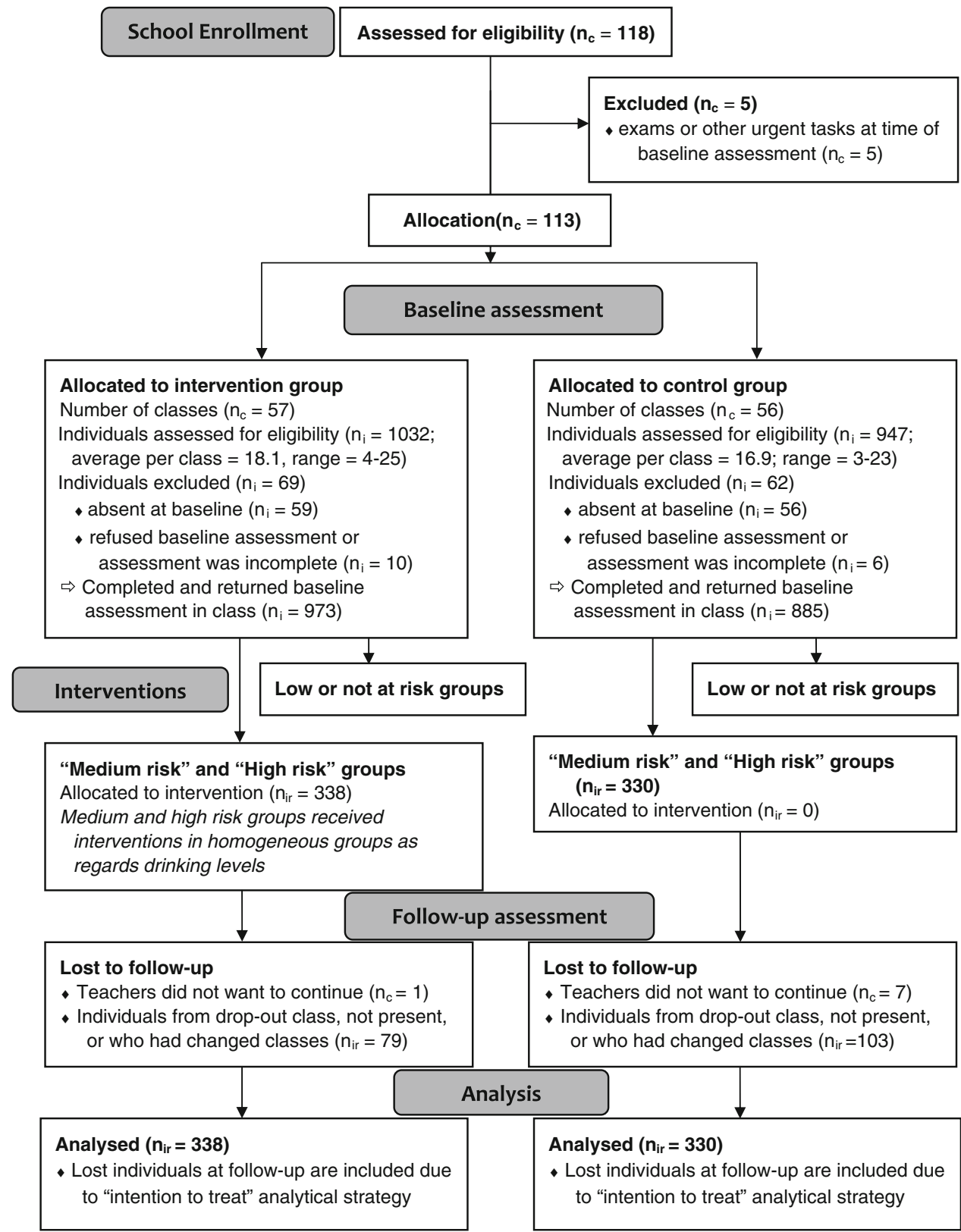

$\mathrm{n}_{\mathrm{c}}=$ number of classes

$\mathrm{n}_{\mathrm{i}}=$ number of individuals

$\mathrm{n}_{\mathrm{ir}}=$ number of individuals in medium risk and high risk groups

Zurich, Switzerland, 2008-2009

these subgroup analyses, which were underpowered to detect differences at the conventional $p<0.05$ level.

\section{Results}

Participant flow and baseline data

Quasi-randomization of classes did not create baseline equivalence on the individual level (Table 2). Students in control classes were significantly older and more often male than those in intervention classes (age and gender were subsequently adjusted). There was no significant difference for risk status $\left(\chi^{2}=1.31, d f=2, p=0.52\right)$ or alcohol outcomes (frequency of RSOD, number of drinks in a typical week or maximum number of drinks on a single occasion).

Seventy-nine individuals from intervention classes and 103 individuals from control classes were lost to follow-up. Since intention to treat was used with the last value carried 
Table 2 Baseline characteristics at cluster (class) level, including students not at risk

\begin{tabular}{|c|c|c|c|c|}
\hline & Intervention & Control & $\begin{array}{l}\text { Test } \\
\text { values }\end{array}$ & $p$ value \\
\hline$n$ & 973 & 885 & & \\
\hline Age, mean $(\mathrm{SD})^{\mathrm{a}}$ & $16.9(1.7)$ & $17.5(2.2)$ & -6.74 & $<0.001$ \\
\hline$\%$ male $^{\mathrm{b}}$ & 47.3 & 55.6 & 12.83 & $<0.001$ \\
\hline $\begin{array}{l}\text { Risk groups: overall } \\
\text { Chi-square }^{\mathrm{c}}\end{array}$ & & & 1.31 & 0.520 \\
\hline$\%$ no risk/low risk & 65.3 & 62.7 & & \\
\hline$\%$ medium risk & 15.8 & 17.0 & & \\
\hline$\%$ high risk & 18.9 & 20.3 & & \\
\hline $\begin{array}{l}\text { \# RSOD past } 30 \text { days; } \\
\text { among RSO drinkers, } \\
\text { mean }(\mathrm{SD})^{\mathrm{a}}\end{array}$ & $3.9(3.1)$ & $4.0(3.1)$ & -0.56 & 0.579 \\
\hline $\begin{array}{l}\text { \# drinks per typical week } \\
\text { past } 30 \text { days; drinkers } \\
\text { only, mean }(\mathrm{SD})^{\mathrm{a}}\end{array}$ & $11.8(14.1)$ & $12.1(16.2)$ & -0.31 & 0.756 \\
\hline $\begin{array}{l}\text { \# maximum drinks on an } \\
\text { occasion past } 30 \text { days; } \\
\text { drinkers only, mean } \\
(\mathrm{SD})^{\mathrm{a}}\end{array}$ & $7.6(4.1)$ & $7.5(4.2)$ & 0.47 & 0.640 \\
\hline
\end{tabular}

Zurich, Switzerland, 2008-2009

$R S O D$ risky single occasion drinking (occasions with $4+$ drinks for female and $5+$ drinks for male students)

${ }^{\mathrm{a}} t$ test: $d f=1,856$ for total study population; $d f=1,233$ for RSO drinkers only; $d f=1,545$ for drinkers only

b Chi-square test $(d f=1)$

c Chi-square test $(d f=2)$

forward, the analysis included 338 at-risk students of 973 $(34.7 \%)$ students in intervention classes and 330 (37.3\%) of the 885 students in control classes.

Attrition analysis (Table 3) showed that individuals dropping out were significantly more likely to be at risk, but there was no differential dropout between intervention and control groups (Wald test for interaction $=0.01$, $p=0.945)$. Among at-risk students, attritors were more likely to be older $(F=4.89, p=0.027)$ and to have more frequent $\operatorname{RSOD}(F=5.67, p=0.018)$, but attrition overall was not differential $(p>0.10)$ regarding sex, number of drinks in a typical week, or maximum drinks on a single occasion.

\section{Main and secondary outcomes}

Although the intervention and the control groups reduced their drinking (Table 4), no significant change was observed for the main outcome of RSOD frequency ( $b=-0.210$, robust $\mathrm{SE}=0.263, p=0.427)$. Similarly, no significant changes were obtained for the secondary outcomes of number of drinks in a typical week $(b=0.534$, robust $\mathrm{SE}=1.134, p=0.639)$ and maximum

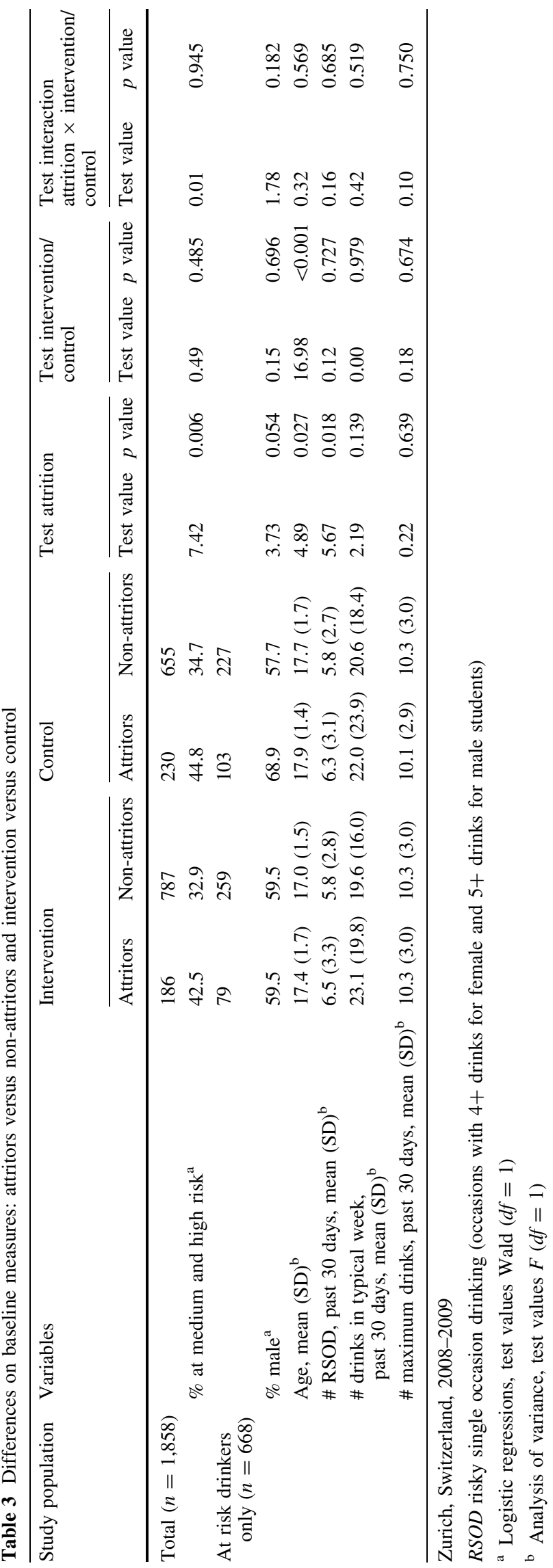


Table 4 Drinking outcome difference from baseline to follow-up by intervention groups (ITT)

\begin{tabular}{|c|c|c|c|c|c|c|c|c|}
\hline & Baseline & Follow-up & Difference & Coeff. & Robust SE & $p$ value & $95 \%$ CI lower & $95 \%$ CI upper \\
\hline \multicolumn{9}{|c|}{ \# RSOD past 30 days, mean (SD) } \\
\hline Intervention & $5.9(3.0)$ & $4.9(3.4)$ & $-1.0(2.9)$ & -0.210 & 0.263 & 0.427 & -0.732 & 0.312 \\
\hline Control & $6.0(2.9)$ & $5.1(3.5)$ & $-0.9(3.1)$ & & & & & \\
\hline \multicolumn{9}{|c|}{ \# drinks in typical week past 30 days, mean (SD) } \\
\hline Intervention & $20.4(17.0)$ & $19.7(17.9)$ & $-0.7(13.6)$ & 0.534 & 1.134 & 0.639 & -1.714 & 2.782 \\
\hline Control & $21.0(20.2)$ & $19.8(19.8)$ & $-1.2(15.0)$ & & & & & \\
\hline \multicolumn{9}{|c|}{ \# maximum drinks on one occasion past 30 days, mean (SD) } \\
\hline Intervention & $10.3(3.0)$ & $9.7(3.6)$ & $-0.6(3.2)$ & 0.190 & 0.236 & 0.423 & -0.278 & 0.658 \\
\hline Control & $10.3(3.0)$ & $9.6(3.6)$ & $-0.7(3.2)$ & & & & & \\
\hline
\end{tabular}

Zurich, Switzerland, 2008-2009

Tests were adjusted for age and sex; analyses among medium risk and high risk groups $(n=668)$

$I T T$ intention to treat analysis; for individuals lost to follow-up the last value forward approach was used, $R S O D$ risky single occasion drinking (occasions with $4+$ drinks for female and $5+$ drinks for male students)

number of drinks on one occasion $(b=0.190$, robust $\mathrm{SE}=0.236, p=0.423)$. The same was true for the analysis stratified by sex (not tabulated); results were highly non-significant $(p>0.4)$ for both men and women.

Ancillary analysis

Analyses stratified by risk groups revealed differential results (Table 5). Beneficial effects of intervention were found for the medium risk group; however, they reached only borderline significance for frequency of RSOD $(b=-0.553$, robust $\mathrm{SE}=0.295, p=0.064)$ and number of drinks in a typical week in the past 30 days $(b=$ -2.824 , robust $\mathrm{SE}=1.480, p=0.059$ ). This represents a 14 and $17 \%$ reduction (respectively) over that of the control group. In gender-specific analyses (not tabulated), effects were in the same direction for males and females. Reduction of RSOD was significant only for females $(b=-0.753, \quad \mathrm{SE}=0.307, \quad p=0.017)$, whereas only males showed reductions in number of drinks in a typical week $(b=-4.403, \mathrm{SE}=2.178, p=0.047)$. Gender differences were non-significant $(p>0.4)$ for maximum number of drinks. After Bonferroni corrections for multiple testing, none of these effects were even of borderline significance, except for RSOD among women.

Changes among the high-risk group were in the opposite direction; controls had greater reductions in consumption compared with the intervention group. Effects were significant for number of drinks in a typical week $(b=3.488$, robust $\mathrm{SE}=1.698, p=0.042$ ), and reached borderline significance for maximum number of drinks on one occasion $(b=0.537$, robust $\mathrm{SE}=0.306, p=0.083)$. Genderspecific analyses (not tabulated) were in the same direction for both genders but revealed a significant increase in number of drinks in a typical week only for males $(b=4.980, \mathrm{SE}=2.410, p=0.042)$ and a borderline increase in maximum number of drinks only for males $(b=0.592, \mathrm{SE}=0.342, p=0.087)$. RSOD frequency remained virtually the same for control and intervention groups, and effects were non-significant $(p>0.4)$ in analyses stratified by gender. None of these results were significant after Bonferroni corrections.

\section{Discussion}

The present study is one of the few using brief alcohol interventions delivered to groups of students. The research aimed to provide evidence for the usefulness of this type of intervention. In Switzerland, evidence on feasibility and effectiveness is required before implementation of largescale interventions. To demonstrate feasibility, the intervention was set up in a way that can be repeated in all schools. It was not designed as a "laboratory" trial to increase internal validity, e.g. setting up the intervention site outside of regular class times and rooms, or using additional research staff or resources such as laptops for data collection. Unfortunately, the unsolicited participation of schools was necessary to provide feasibility evidence in the current trial, and certain standards for state-of-the-art randomized clinical efficacy trials could not be met (e.g. random selection of schools or perfect random allocation of classes to control and intervention conditions). We believe that these shortcomings were counteracted by showing that these interventions can be accomplished within the standard curriculum. Therefore, external validity is high, but can only be generalized to secondary schools in the German-speaking Zürich Canton.

The findings of the present study went in different directions. Strictly speaking, there were no significant 
Table 5 Drinking outcomes differences between baseline and follow-up time by intervention groups and at risk subgroups (ITT)

\begin{tabular}{|c|c|c|c|c|c|c|c|c|}
\hline & Baseline & Follow-up & Difference & Coeff. & Robust SE & $p$ value & $95 \%$ CI lower & $95 \%$ CI upper \\
\hline \multicolumn{9}{|c|}{ \# RSOD past 30 days, mean (SD) } \\
\hline \multicolumn{9}{|l|}{ Medium risk } \\
\hline Intervention & $3.5(0.0)$ & $3.1(2.3)$ & $-0.4(2.3)$ & -0.553 & 0.295 & 0.064 & -1.138 & 0.032 \\
\hline Control & $3.5(0.0)$ & $3.6(2.7)$ & $0.1(2.7)$ & & & & & \\
\hline \multicolumn{9}{|l|}{ High risk } \\
\hline Intervention & $8.0(2.6)$ & $6.4(3.4)$ & $-1.6(3.2)$ & 0.055 & 0.363 & 0.881 & -0.666 & 0.775 \\
\hline Control & $8.0(2.4)$ & $6.3(3.7)$ & $-1.7(3.2)$ & & & & & \\
\hline \multicolumn{9}{|c|}{ \# drinks in typical week past 30 days, mean (SD) } \\
\hline \multicolumn{9}{|l|}{ Medium risk } \\
\hline Intervention & $15.5(13.5)$ & $13.6(11.1)$ & $-1.9(11.9)$ & -2.824 & 1.480 & 0.059 & -5.762 & 0.113 \\
\hline Control & $13.2(9.0)$ & $13.9(12.7)$ & $0.7(11.0)$ & & & & & \\
\hline \multicolumn{9}{|l|}{ High risk } \\
\hline Intervention & $24.6(18.5)$ & $24.7(20.8)$ & $0.1(14.9)$ & 3.488 & 1.698 & 0.042 & 0.121 & 6.855 \\
\hline Control & $27.6(24.3)$ & $24.6(23.1)$ & $-3.0(17.4)$ & & & & & \\
\hline \multicolumn{9}{|c|}{ \# maximum drinks on one occasion past 30 days, mean (SD) } \\
\hline \multicolumn{9}{|l|}{ Medium risk } \\
\hline Intervention & $9.4(3.3)$ & $8.7(3.8)$ & $-0.7(3.4)$ & -0.202 & 0.358 & 0.573 & -0.912 & 0.507 \\
\hline Control & $9.1(3.2)$ & $8.7(3.7)$ & $-0.5(3.2)$ & & & & & \\
\hline \multicolumn{9}{|l|}{ High risk } \\
\hline Intervention & $11.2(2.4)$ & $10.5(3.1)$ & $-0.7(3.1)$ & 0.537 & 0.306 & 0.083 & -0.070 & 1.145 \\
\hline Control & $11.2(2.5)$ & $10.2(3.4)$ & $-1.0(3.3)$ & & & & & \\
\hline
\end{tabular}

Zurich, Switzerland, 2008-2009

Tests were adjusted for age and sex; medium risk: 3-4 occasions of RSOD in the past 30 days; high risk: 5 or more occasions of RSOD in the past 30 days

ITT intention to treat; for individuals lost to follow-up the last value forward approach was used, $R S O D$ risky single occasion drinking (occasions with $4+$ drinks for female and $5+$ drinks for male students)

results for at risk-drinkers taken as a whole. However, there were divergent findings for 'medium risk' (3-4 RSOD) and 'high risk' drinkers (5 or more RSOD). The intervention yielded consistent reductions in alcohol use for medium risk group, reaching borderline significance $(p<0.10)$ for the main outcome of RSOD frequency and the secondary outcome of number of drinks in a typical week. Reductions were about $15 \%$ greater than in the control group and are consistent with effect sizes described in BAI meta-analyses (Bertholet et al. 2005) and other school-based prevention programs (Faggiano et al. 2010). However, it should be noted that after Bonferroni corrections for multiple testing nothing was significant at the nominal $p<0.05$ alpha level.

Alcohol use within the high risk group seemed to increase following BAI compared with controls and was significant for number of drinks in a typical week $(p<0.05)$, and borderline significant for maximum number of drinks on an occasion $(p<0.10)$. Virtually no differences were found for frequency of RSOD.

Generally, a conclusion of the BAI literature is that there are either beneficial or null effects, but none that are detrimental (Larimer et al. 2004; Tevyaw and Monti 2004;
Handmaker et al. 1999; Murphy et al. 2004). Although it is unlikely that group BAI will produce detrimental effects, the present study suggests using caution regarding highrisk subgroups.

An explanation for some apparent detrimental effects on high-risk drinkers has been given by McCambridge et al. (2011). Responses to alcohol use questionnaires are subject to different biases, e.g. social desirability (e.g. Davis et al. 2010), faulty recall (Gmel and Daeppen 2007), or assessment reactivity (Walters et al. 2009). It may be that heavy drinkers "learn" from feedback during the intervention that others also have equally high (or higher) consumption levels. At follow-up, they may be more prone to revealing their "true" consumption (reduction of social desirability bias), to remember more frequent or heavier drinking (reduction in recall bias) or to assess their consumption levels more accurately (assessment reactivity). Two observations support this argument. First, negative effects were found for number of drinks during a typical week and maximum drinks on an occasion, but not for frequency of RSOD, which occurs mostly on weekends with regularity and is probably easier to remember. RSOD frequency may 
also be less socially undesirable to report (e.g. "everybody drinks on weekends") than the actual amounts consumed during these occasions. Second, counselors often alluded to this phenomenon by reporting that during the first intervention session, self-reported alcohol levels were lower. By the second session, students had more confidence and were more open in their discussions with others, and admitted drinking more heavily on some occasions. If these mechanisms were actually in play, then more accurate selfreporting at follow-up may have masked any real reductions in alcohol use due to the intervention.

Drawing conclusions from this trial depends on the interpretation of negative effects obtained in the "high risk" group. Babor and Higgins-Biddle (2001) have noted that BAI is not designed for individuals with very heavy drinking, and many BAI trials either have excluded the heaviest drinkers or (if included) have found lower effect sizes (Kaner et al. 2007). It may be that BAI does not work well on heavy drinking secondary school students. There is also some discussion in the literature regarding the potential for iatrogenic effects of group interventions (Dishion et al. 2002). However, a meta-analysis was not supportive of iatrogenic or deviancy training effects (Weiss et al. 2005). If there is truly a case for iatrogenic effects, then group BAI should not be recommended without incorporating dedicated measures for heavy drinking groups into the intervention design. Model programs have been advanced by organizations such as the U.S. Substance Abuse and Mental Health Services Administration (SAMHSA), e.g. Active Parenting of Teens, Motivational Enhancement Therapy, Adolescent Community Reinforcement Approach, Multidimensional Family Therapy or Twelve Step Facilitation Therapy. These programs go far beyond the BAI of the present study and require a lot more effort and additional resources. Although BAI should not be seen as an alternative to such programs or a replacement for alcohol education in early age, it may add to the spectrum of possible interventions at relatively low expense.

One-on-one interventions have the advantage of providing a personalized and confidential setting in which counselors can focus on an individual's change talk to stimulate behavioral change (Miller and Rollnick 2002). In contrast, group interventions have greater cost-effectiveness and the ability for implementation in secondary schools. A larger majority of younger students can be reached without introducing undesirable "side effects", such as stigmatization by classmates or teachers. Several theoretical arguments can also be made for group interventions, such as direct peer feedback (LaBrie et al. 2007), reinforcement by peers that strengthens autonomy within the group (Foote et al. 1999), and enhancement of autonomous motivation (Ryan et al. 1995). On the other hand, social dynamics in the classroom might repress openness among shy students. Feedback of counselors, however, confirmed some very lively discussions among participants, and shy students may nevertheless have benefitted from these discussions even though they seldom joined in. Our study showed that group BAI may be beneficial for some heavy drinkers, but may be detrimental for the heaviest of them. To scientifically establish that benefits without harm exist, designs are needed that can test for reduction of social desirability and recall biases or assessment reactivity in group sessions affecting selfreports of consumption. However, if the apparent increase in alcohol use within high-risk students shown here is confirmed, then group BAI either should be discontinued or at least modified for heavy drinkers.

\section{References}

Babor TF, Higgins-Biddle JC (2001) Brief intervention for hazardous and harmful drinking: a manual for use in primary care. In: World Health Organization (WHO), Department of Health and Substance Department (ed) Screening and brief intervention: for alcohol problems in primary care, 2nd edn. WHO, Geneva

Babor TF, Caetano R, Casswell S, Edwards G, Giesbrecht N, Graham $\mathrm{K}$, Grube JW, Hill L, Holder HD, Homel R, Livingston M, Österberg E, Rehm J, Room R, Ingeborg R (2010) Alcohol: no ordinary commodity: research and public policy. Oxford University Press, Oxford

Bachmann K (1999) Lust oder Last-Berufszufriedenheit und Belastung im Beruf bei Lehrerinnen und Lehrern an berufsbildenden Schulen. Schneider Verlag Hohengehren GmbH, Baltmannsweiler

Bertholet N, Daeppen J-B, Wietlisbach V, Fleming M, Burnand B (2005) Reduction of alcohol consumption by brief alcohol intervention in primary care: systematic review and meta-analysis. Arch Intern Med 165(9):986-995. doi:10.1001/archinte.165.9.986

Bland JM (2000) Sample size in guidelines trials. Fam Pract 17(Suppl 1):S17-S20

Bradley AC, Baker A, Lewin TJ (2007) Group intervention for coexisting psychosis and substance use disorders in rural Australia: outcomes over 3 years. Aust N Z J Psychiatry 41(6):501-508. doi:10.1080/00048670701332300

D'Amico EJ, Osilla KC, Hunter SB (2010) Developing a group motivational interviewing intervention for adolescents at-risk for developing an alcohol or drug use disorder. Alcohol Treat Quart 28(4):417-436. doi:10.1080/07347324.2010.511076

Davis CG, Thake J, Vilhena N (2010) Social desirability biases in self-reported alcohol consumption and harms. Addict Behav 35(4):302-311. doi:10.1016/j.addbeh.2009.11.001

Dishion TJ, Bullock BM, Granic I (2002) Pragmatism in modeling peer influence: dynamics, outcomes, and change processes. Dev Psychopathol 14(4):969-981

Engle B, Macgowan MJ, Amrhein PC (2010) Markers of marijuana use outcomes within adolescent substance abuse group treatment. Res Soc Work Prac 20(3):271-282. doi:10.1177/1049731 509347855

Ezzati M, Lopez AD, Rodgers A, Murray CJL (2004) Comparative quantification of health risks. Global and regional burden of disease attributable to selected major risk factors, vol 1 and 2 . World Health Organization (WHO), Geneva 
Faggiano F, Vigna-Taglianti F, Burkhart G, Bohrn K, Cuomo L, Gregori D, Panella M, Scatigna M, Siliquini R, Varona L, van der Kreeft P, Vassara M, Wiborg G, Galanti MR (2010) The effectiveness of a school-based substance abuse prevention program: 18-month follow-up of the EU-Dap cluster randomized controlled trial. Drug Alcohol Depend 108(1-2):56-64. doi: 10.1016/j.drugalcdep.2009.11.018

Fields A (2006) Resolving patient ambivalence. A five session motivational interviewing intervention. Hollifield Associates, Washington

Foote J, DeLuca A, Magura S, Warner A, Grand A, Rosenblum A, Stahl $S$ (1999) A group motivational treatment for chemical dependency. J Subst Abuse Treat 17(3):181-192 pii:S0740547299000033

Gmel G, Daeppen JB (2007) Recall bias for seven-day recall measurement of alcohol consumption among emergency department patients: implications for case-crossover designs. J Stud Alcohol Drugs 68(2):303-310

Gmel G, Kuntsche E, Rehm J (2011) Risky single-occasion drinking: bingeing is not bingeing. Addiction 106(6):1037-1045. doi: 10.1111/j.1360-0443.2010.03167.x

Handmaker NS, Miller WR, Manicke M (1999) Findings of a pilot study of motivational interviewing with pregnant drinkers. J Stud Alcohol Drugs 60(2):285-287

Hibell B, Adlaf EM, Andersson B, Bjarnason T, Delapenha C, Hasbun J, Johnston L, Sathianathan R (2003) Conducting school surveys on drug abuse. Toolkit module 3. United Nations Office on Drugs and Crime, Vienna, Austria

Hingson RW, Heeren T, Winter MG, Wechsler H (2005) Magnitude of alcohol-related mortality and morbidity among U.S. college students ages 18-24: changes from 1998 to 2001. Annu Rev Public Health 26:259-279

Hintze JL (ed) (2008) PASS 2008 User's Guide. NCSS, Dr. Jerry L. Hintze, Kaysville, UT

Kaner EF, Beyer F, Dickinson HO, Pienaar E, Campbell F, Schlesinger C, Heather N, Saunders J, Burnand B (2007) Effectiveness of brief alcohol interventions in primary care populations. Cochrane Database Syst Rev 2:CD004148. doi: 10.1002/14651858.CD004148.pub3

LaBrie JW, Pedersen ER, Lamb TF, Quinlan T (2007) A campusbased motivational enhancement group intervention reduces problematic drinking in freshmen male college students. Addict Behav 32(5):889-901. doi:10.1016/j.addbeh.2006.06.030

LaBrie JW, Huchting KK, Lac A, Tawalbeh S, Thompson AD, Larimer ME (2009) Preventing risky drinking in first-year college women: further validation of a female-specific motivational-enhancement group intervention. J Stud Alcohol Suppl 16:77-85

LaChance H, Feldstein Ewing SW, Bryan AD, Hutchison KE (2009) What makes group MET work? A randomized controlled trial of college student drinkers in mandated alcohol diversion. Psychol Addict Behav 23(4):598-612. doi:10.1037/a0016633

Larimer ME, Cronce JM, Lee CM, Kilmer JR (2004) Brief intervention in college settings. Alcohol Res Health 28(2):94-104

Lumley T, Diehr P, Emerson S, Chen L (2002) The importance of the normality assumption in large public health data sets. Annu Rev Public Health 23:151-169. doi:10.1146/annurev.publhealth.23. 100901.140546
Lundahl B, Burke BL (2009) The effectiveness and applicability of motivational interviewing: a practice-friendly review of four meta-analyses. J Clin Psychol 65(11):1232-1245. doi:10.1002/ jclp.20638

McCambridge J, Hunt C, Jenkins RJ, Strang J (2011) Cluster randomised trial of the effectiveness of motivational interviewing for universal prevention. Drug Alcohol Depend 114(2-3): 177-184. doi:10.1016/j.drugalcdep.2010.07.028

Miller WR, Rollnick S (2002) Motivational interviewing: preparing people for change, 2nd edn. Guilford Press, New York

Murphy JG, Benson TA, Vuchinich RE, Deskins MM, Eakin D, Flood AM, McDevitt-Murphy ME, Torrealday O (2004) A comparison of personalized feedback for college student drinkers delivered with and without a motivational interview. J Stud Alcohol Drugs 65(2):200-203

Nelson TF, Naimi TS, Brewer RD, Wechsler H (2005) The state sets the rate: the relationship among state-specific college binge drinking, state binge drinking rates, and selected state alcohol control policies. Am J Public Health 95(3):441-446. doi: 10.2105/AJPH.2004.043810

Petitti DB (2000) Meta-analysis, decision analysis, and cost-effectiveness analyses. Methods for quantitative synthesis in medicine, 2nd edn. Oxford University Press, New York

Rehm J, Taylor B, Room R (2006) Global burden of disease from alcohol, illicit drugs and tobacco. Drug Alcohol Rev 25(6):503513. doi:10.1080/09595230600944453

Roberts C, Tynjälä J, Currie D, King M (2004) Annex 1. Methods. In: Currie C, Roberts C, Morgan A et al (eds) Young people's health in context-health behaviour in school-aged children (HBSC) study: International Report from the 2001/2002 Survey. World Health Organization (WHO), Regional Office for Europe, Copenhagen, pp 217-227

Ryan RM, Plant RW, O'Malley S (1995) Initial motivations for alcohol treatment: relations with patient characteristics, treatment involvement, and dropout. Addict Behav 20(3):279-297 pii:0306-4603(94)00072-7

Schmiege SJ, Broaddus MR, Levin M, Bryan AD (2009) Randomized trial of group interventions to reduce HIV/STD risk and change theoretical mediators among detained adolescents. J Consult Clin Psychol 77(1):38-50. doi:10.1037/a0014513

Tevyaw TO, Monti PM (2004) Motivational enhancement and other brief interventions for adolescent substance abuse: foundations, applications and evaluations. Addiction 99(Suppl 2):63-75. doi: 10.1111/j.1360-0443.2004.00855.x

Walters ST, Vader AM, Harris TR, Jouriles EN (2009) Reactivity to alcohol assessment measures: an experimental test. Addiction 104(8):1305-1310. doi:10.1111/j.1360-0443.2009.02632.x

Weiss B, Caron A, Ball S, Tapp J, Johnson M, Weisz JR (2005) Iatrogenic effects of group treatment for antisocial youths. J Consult Clin Psychol 73(6):1036-1044. doi:10.1037/0022006X.73.6.1036

Whitlock EP, Polen MR, Green CA, Orleans T, Klein JD (2004) Behavioral counseling interventions in primary care to reduce risky/harmful alcohol use by adults: a summary of the evidence for the U.S. Preventive Services Task Force. Ann Intern Med 140 (7):557-568. pii:140/7/557 